\section{Diffusionslänge der Ladungsträger in CdS}

\author{
Von J. A u th und E. A. Niekis ch
}

II. Physikalisches Institut der Humboldt-Universität, Berlin (Z. Naturforschg. 10 a, 1035 [1955] ; eingegangen am 24. Oktober 1955)

Ein photoleitender CdS-Kristall sei auf seiner Oberfläche mit zwei sperrschichtfreien „ohmschen“ Elektroden im Abstand $L$ versehen (Spaltzelle, Abb. 1). Er werde im Gebiet $\left(x_{0}, L\right)$ mit einer konstanten Intensität beleuchtet; von dem Gebiet $\left(0, x_{0}\right)$ werde durch eine (verschiebbare) Blende das Licht ferngehalten. Die Farbe des Lichts sei so, daß es vollständig im Kristallinnern absorbiert werde $(\lambda<5000 \AA)$. Die im belichteten Teil des Kristalls erzeugten Elektronen und Löcher diffundieren in den dunklen Teil; zusammen mit Rekombination und entstehender Diffusionsspannung werden sich stationäre Ladungsträgerkonzentrationen $n(x)$ und $p(x)$ (Elektronen bzw. Löcher) ausbilden. Wir beschreiben eine einfache Methode zur Messung von $n(x)$.

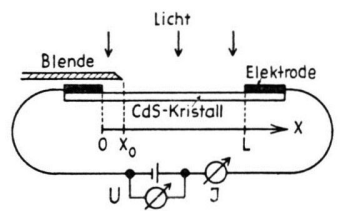

Abb. 1.

Wir beschränken uns auf eine eindimensionale Behandlung des Problems und benutzen den Umstand, daß CdS ein ausgesprochener Überschußleiter ist. Durch den Kristall fließt dann ein Strom mit der Stromdichte

$$
j=e n(x) b_{\mathrm{n}} E(x)+e D_{\mathrm{n}} \cdot \mathrm{d} n(x) / \mathrm{d} x .
$$

Natürlich hängen $j, n(x)$ und $E(x)$ auch von der Stellung der Blende, d. h. von dem Parameter $x_{0}$ ab. Wir lösen (1) nach der Feldstärke $E(x)$ auf und integrieren über die Spaltbreite $L$. Diese „Bahnspannung“ hängt mit der von außen an den Kristallelektroden liegenden Spannung $U$ wie folgt zusammen:

$$
U=\left(j / e b_{\mathrm{n}}\right) \int_{0}^{L} \mathrm{~d} x / n(x)-\left(D_{\mathrm{n}} / b_{\mathrm{n}}\right) \ln (n(L) / n(0))+\Delta .
$$

Dabei entspricht das Integral dem ohmschen Bahnwiderstand, das zweite Glied ist die Diffusionsspannung und der dritte Term $\Delta$ berücksichtigt die an den Grenzen Halbleiter - Elektrodenmetall auftretenden Potentialsprünge; $\Delta$ ist deren Differenz. Man kann an Hand der „Quasi-Fermi-Grenze“ der Elektronen und der der Defektelektronen zeigen, daß bei einem $\mathrm{n}$-Leiter wie
CdS sich $\Delta$ und der Diffusionsterm weitgehend gegeneinander kompensieren. Dies steht in Übereinstimmung mit dem experimentellen Befund, da $\beta$ im stromlosen Falle keine Photospannung nachgewiesen werden konnte (Meßgenauigkeit $\sim 10^{-3}$ Volt) .

Eine der möglichen Varianten unseres Versuchs besteht nun darin, bei einer Änderung der Blendenstellung $x_{0}$ die Spannung so zu ändern, daß der Strom konstant bleibt (man darf die Spannungen natürlich nicht so groß machen, daß sich dadurch $n(x)$ merklich ändert). Dann ist, mit Berücksichtigung des vorhin Gesagten

$$
U\left(x_{0}\right) \approx\left(j / e b_{\mathrm{n}}\right) \int_{0}^{L} \mathrm{~d} x / n\left(x, x_{0}\right) .
$$

Es hängt aber die Konzentrationsverteilung der Ladungsträger nur von der relativen Lage zur Blende $a b, d . h$. es ist $n\left(x, x_{0}\right)=n\left(x_{0}-x\right)$. Durch eine Differentiation nach $x_{0}$ kann man somit das Integral über $x$ beseitigen und man bekommt (mit einer unbedeutenden Vernachlässigung)

$$
\mathrm{d} U / \mathrm{d} x_{0}=\left(j / e b_{\mathrm{n}}\right)\left(1 / n\left(x_{0}\right)-1 / n_{0}\right)
$$

$\left(n_{0}\right.$ Elektronenkonzentration im belichteten Teil des Kristalls), so daß man also nach einer graphischen Differentiation der Meßkurven $U=U\left(x_{0}\right)$ aus (4) $n\left(x_{0}\right)$ entnehmen kann (Abb. 2). Erste Messungen ergeben Diffusionslängen von 1 bis $3 \cdot 10^{-3} \mathrm{~cm}$ bei „reinen“ (nicht bewußt aktivierten) Kristallen.

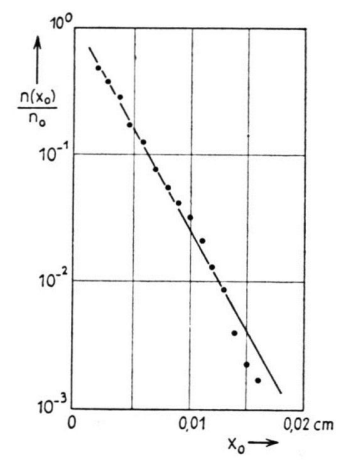

Abb. 2.

Wir nehmen an, daß wir es mit „ambipolarer“ Diffusion zu tun haben; die erhaltene Diffusionslänge enthält also im wesentlichen die Diffusionskonstante der Löcher.

Für eine Einstrahlung im Bereich der Grundgitterabsorption folgt aus unserem Ergebnis, daß der leitende Bereich etwa 100-mal breiter ist als die Schicht, innerhalb der das anregende Licht absorbiert wird $\left(\sim 10^{-5} \mathrm{~cm}\right)$. 